\title{
Position statement Euromed Network on Mental Health and Migration
}

\author{
Adil Qureshi', Driss Moussaoui \\ From $1^{\text {st }}$ International Congress on Neurobiology and Clinical Psychopharmacology and European \\ Psychiatric Association Conference on Treatment Guidance \\ Thessaloniki, Greece. 19-22 November 2009
}

\section{Introduction/context}

Migration is one of the most important challenges of the XXIst century. Europe in particular has seen a dramatic rise in migration over the past decade, and all indications suggest that this increase will continue well into the future.

It is becoming increasingly clear that the mental health needs of migrants require specific attention. Recent reports indicate that migrants are underrepresented in mental health care centers, are subject to more diagnostic error, more coercive measures and involuntary treatment, less psychotherapy and less second generation medication [1,2]. The specific relationship between migration and mental health remains unclear, although a number of protective and risk factors have been identified [3]. What would appear to be clear is that there is a robust relationship between migration and schizophrenia, with odds rations varying from 2 to 8 for individuals from the European community for the former and Afrocaribbean migrants for the latter [4]. In addition, research indicates that the children of immigrants-second generation citizens-also have higher odds ratios for the development of psychotic and affective disorders.

All of this has implications for psychology and psychiatry as a whole. The field of transcultural psychiatry overtly emphasizes the biopsychosocial model: that migration overwhelmingly impacts the incidence of schizophrenia emphasizes that experience itself, and not simply biological structure or genetics is involved. Difficulties with diagnosis demand reconsideration of existing nosological systems, as reflected, for example, in the pathway towards the DSM-V and the ICD-11, and treatment issues, be they psychotherapeutic or

Euromed Network on Mental Health and Migration pharmacological indicate that treatments may need to be adapted given the cultural and/or ethnic background and migratory experience of the patient $[5,6]$.

A clear conclusion is that the mental health needs of immigrants are not being met [7]. This can be seen at the two levels identified above and are related to both prevention and treatment. To that end the Euromed network calls for the development of both clinical and institutional cultural competence by attending to research, training, and advocacy. This human rights issue which involves considerable suffering implicates all levels of stakeholders, from individuals to their families, from clinicians to the institutions they work for, to health care systems, professional associations, and professional training programs.

\section{Research}

Most all of what we know about migration and mental health comes from research. Although considerable strides have been made in this area, it is clear that there remains much to be done. We call for more research to be facilitated and carried out in this area. It is noteworthy that the vast bulk of psychological and psychiatric research uses a "gold standard" that is not representative of more than $80 \%$ of the world's population, which means that much of what we know may be of questionable applicability to the latter, which is particularly true of clinical trials.

Indeed, the field of ethnopsychopharmacology clearly demonstrates that pharmacokinetics and pharmacodynamics are not "universal" but rather vary with the genetic makeup [6]. Thus treatment, be it pharmacological or psychological, needs to be investigated at more population specific levels in order to ensure that all patients receive an optimal quality of care. 
It is essential that research is carried out on all aspects related to the improvement of care of migrants, from pharmacological intervention to psychotherapy to the participation of intercultural mediators and medical interpreters. In addition, research needs to examine institutional and administrative characteristics to determine what maximizes access and quality of care. All of this is necessary to improve the overall cultural competence of the mental health care system.

One of the big questions facing researchers has to do with the degree to which immigration in and of itself constitutes a risk factor for common mental disorders, on the one hand, and what best explains the relationship between immigration and schizophrenia [4]. In addition to requiring further exploration, these areas of research all thematize the complexity of applying research methodology and instrumentation developed in one particular cultural, social, and political context in another. Psychometrics are clearly influenced by cultural differences, to the extent that leading experts agree that there is no such thing as a "culture free" or even "culture fair" test [8]. This means that how research is carried out needs to be rethought, with, perhaps, more emphasis given to qualitative approaches.

The area of prevention also warrants attention. Clearly, the optimal way of improving the mental health of a population is to prevent mental health problems and promote mental health. How to do this, however, demands a better understanding of the risk and protective factors related to the migratory process. Research in this area is growing and needs to be further developed and then made relevant to mental health promotion and prevention and treatment on psychopathology.

\section{Training and education}

In today's increasingly diverse world, it is arguable that culture and difference play a role in most every sort of patient contact. To that end, it is, in our opinion, an ethical requirement that all mental health professionals receive training in cultural competence. The very notion of professional competence is predicated on a combination of practical experience along with theory and research [9]. Whereas many clinicians have ample experience working with migrants, they all too often lack a scientific basis on which to frame and further develop their work.

Training should be focused on promotion of mental health, prevention of psychopathology, and diagnosis and treatment, and should be provided not only to clinicians but also to administrators, researchers, as well as all clinical staff.

We need to have a better understanding of the effectiveness of training. Many models exist; however, there exists minimal research that evaluates the clinical impact. We need to know what sorts of training initiatives have a real world impact on the sort of care imparted by trainees.

\section{Advocacy}

As things stand, it is clear tha migrants are not having their best interests attended to. It is essential that those with the means to do so advocate on behalf of this more vulnerable population ranging from advocacy at the community to that of the individual level.

In a related vein, we call upon national and international associations to organize or promote sections or special interest groups related to migration and mental health/transcultural psychiatry as a means of identifying and needs and developing appropriate responses. Such special interest groups can then network in order to further share ideas, experiences, and research findings, with an eye to improving the mental health of migrants.

\section{The Euromed Network on Migration and Mental Health}

One of the central objectives of the Euromed Network on Migration and Mental Health is the development of a forum in which local and relevant research, experiences, and initiatives can be shared, compared, and contrasted with the objective of contributing to an overall improvement in the mental health of migrants.

Published: 22 April 2010

\section{References}

1. Nagayama Hall GC: Psychotherapy research with ethnic minorities: Empirical, ethical, and conceptual issues. J Consulting Clin Psychol 2001, 69(5):502-510.

2. Lay B, Lauber $C$, ouml, ssler W: Are immigrants at a disadvantage in psychiatric in-patient care? Acta Psychiatrica Scandinavica 2005, 111:358-366.

3. Bhugra D: Migration and mental health. Acta Psychiatrica Scandinavica 2004, 109(4):243-258.

4. Cantor-Graae E, Selten JP: Schizophrenia and migration: A metal-analysis and review. Am J Psychiatry 2005, 162(1):12-24.

5. Sue S: In defense of cultural competency in psychotherapy and treaetment. Am Psychol 2003, 58(11):964-970.

6. Ruiz P: Ethnicity and Psychopharmacology. Ethnicity and Psychopharmacology Washington, DC: American Psychiatric PressRuiz P 2000, 19:91-113.

7. Department of Health: Inside outside: improving mental health services for black and minority ethnic communities in England. London: Department of Health 2003.

8. Vijver van de F, Tanzer NK: Bias and equivalence in cross-cultural assessment. Rev Eur Psychol Appl 2004, 54:119-135.

9. Qureshi A, Collazos F, Ramos M, Casas M: Cultural competency training in psychiatry. Eur Psychiatry 2008, 23(Suppl 1):49-58.

doi:10.1186/1744-859X-9-S1-S237

Cite this article as: Qureshi and Moussaoui: Position statement Euromed Network on Mental Health and Migration. Annals of General Psychiatry 2010 9(Suppl 1):S237. 